\title{
Karriere bei AVL: Antrieb mit System
}

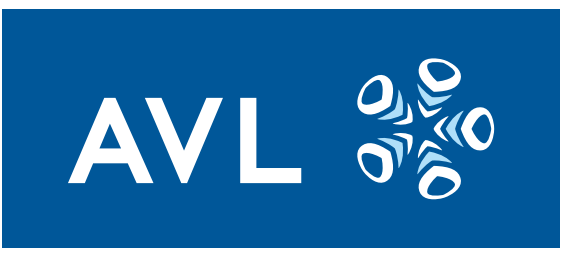

AVL ist als Partner der Fahrzeugindustrie Weltmarktführer in vielen Bereichen. Seit über 60 Jahren liegt die Kernkompetenz von AVL in der Entwicklung neuer Motoren. Dieses Feld hat sich in den letzten 10 bis 15 Jahren deutlich erweitert in Richtung Gesamtantriebssysteme sowie von der Prototypen- hin zur Serienentwicklung.

\section{Entwicklungsingenieur/in und Bauteilverantwortliche/r Serienentwicklung für Graz/Ingolstadt/München/Rüsselsheim/Stuttgart}

Ihr Aufgabenbereich: • Verantwortung für die Bauteile und Entwicklung von Antriebsstrang-Komponenten/Modulen • Prozessverantwortung vom Konzept bis zur Serienproduktion • Fachliche Führung von zugeteilten MitarbeiterInnen • Koordination aller Schnittstellen intern (z. B. Einkauf, Produktionsplanung) und beim Kunden • Lastenhefterstellung (Komponenten und System) sowie versuchstechnische Begleitung der Komponenten/ Module - Abschluss von Leistungsvereinbarungen, intern und extern zur Sicherstellung der Zielerreichung • Steuerung von Systementwicklungslieferanten • Funktions-, Kosten-, Gewichts-, Termin- und Qualitätsverantwortung der Bauteile/Module • Integration der Bauteile in das Gesamtsystem, Vertretung im Simultaneous Engineering Team

Ihr Profil: • HTL oder TU/FH Fachrichtung Maschinenbau/Elektrotechnik/Motoren-/Getriebe-/Fahrzeugtechnik oder vergleichbare bzw. durch Berufserfahrung erworbene Kenntnisse • Einschlägige Berufserfahrung als Bauteilverantwortlicher bzw. Simultaneous Engineering-Teamleiter • Sehr gute Kommunikationsfähigkeit • Selbstständigkeit und Flexibilität • Hohe Einsatzbereitschaft, Verantwortungsbewusstsein sowie Teamfähigkeit • Erste Führungserfahrung sowie sicheres Auftreten bei Präsentationen • Kenntnisse der Kundensysteme/-prozesse von namhaften OEMs erwünscht • Verhandlungssichere Deutsch- und Englischkenntnisse

„Die Herausforderung in der Serienentwicklung lag und liegt nicht nur in der Entwicklung bei AVL, sondern auch bei unseren Kunden und Bauteil- und Systemlieferanten“, so Wolfgang Schöffmann. Immer mehr Hersteller haben sich aus Stückzahl- und Kostengründen zu gemeinsamen Antriebsfamilien zusammengeschlossen. Die entwickelten Motoren und Getriebe werden in unterschiedlichste Fahrzeuge eingebaut. Jeder Hersteller hat oft dutzende

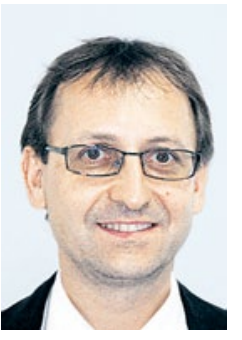

Wolfgang Schöffmann, Leiter PKW-Antriebskonstruktion, Graz
Applikationen, in jedem Fahrzeug sind die Randbedingungen und die Leistungsstufen anders und natürlich auch die Integration eines bereits bestehenden Aggregates in das Fahrzeug. Fachübergreifendes Denken und Handeln ist dabei gefragter denn je, Ingenieurinnen und Ingenieure müssen interdisziplinär denken und arbeiten. Aktuell sucht AVL nach Bauteilverantwortlichen bzw. Modulverantwortlichen. Hier wird ein Simultaneous Engineering Team aufgebaut bzw. ausgebaut, sowohl in Österreich als auch in Deutschland. Es handelt sich dabei um die Schnittstelle zwischen Entwicklung, Produktion, Lieferant und Kunde, es sind Schlüsselpositionen bei Serienprojekten. Prozessverständnis, Organisationstalent und Kommunikationsstärke sind für diese Funktion wichtig. Hier bietet sich ein weites und interessantes Aufgabengebiet in einem internationalen Unternehmen.

Die Niederlassung in Mainz Kastel ist die jüngste Niederlassung im AVL Konzern und befindet sich in der Aufbauphase. Daraus ergeben sich vielfältige Aufgaben und Chancen

mitzugestalten. „Wir unterstützen die Kunden vor Ort und sind Schnittstelle zu allen AVL Tech Centern weltweit mit Schwerpunkten wie Kalibrierung, Konstruktion, Thermodynamik- und Mechanik-Entwicklung, Simulation, Cost Engineering, Projektmanagement, Elektrifikation und Testing." Markus Bungardt ist Niederlassungsleiter und war selber 9 Jahre Bauteilverantwortlicher bei einem großen OEM. Er freut sich über Bewerbungen von Spezia-

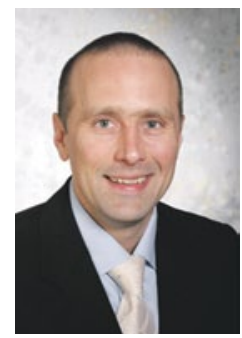

Markus Bungardt, Niederlassungsleiter Mainz Kastel listInnen und jungen Ingenieurlnnen, die diese Herausforderung annehmen wollen.

„Mit unseren vielen, weltweit ansässigen Standorten sehe ich bei AVL noch großes Potenzial für weiteres Wachstum in der Zukunft. Dieses Potenzial erfolgreich zu heben, gelingt uns jedoch nur mit kreativen, engagierten und motivierten Mitarbeiterlnnen. Das Engagement unserer Mitarbeiterlnnen in diesem komplexen Arbeitsumfeld auf diesem hohen Niveau zu halten ist eine unserer spannendsten Aufgaben. Ich selbst habe bei der AVL in der Vergangenheit vielfältige Möglichkeiten

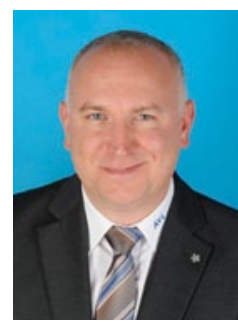

Jan Marek, Niederlassungsleiter Ingolstadt

für meine persönliche Entwicklung aktiv wahrnehmen können und habe immer die gewünschte Unterstützung erhalten. Das sind meine Gründe für 15 Jahre AVL.", so Jan Marek.
Der Trend, die Modellpaletten zu erweitern und gleichzeitig zu aktualisieren, führt neben der notwendigen Anpassung der Kalibrierung der Motor- und Getriebesteuerungen zu erheblichen Entwicklungsumfängen bei Motor- und Getriebehardware. Philipp Milanes erläutert: "AVL passt sich diesen Anforderungen durch einen weiteren Ausbau der Aktivitäten in Stuttgart an. So wird das bereits existierende Engineering Center in der Personalkapazität ausgebaut und um Testeinrichtungen (Rollen-, Antriebsstrang- und Motorenprüfstände) ergänzt. Hier ergeben sich sehr interessante Möglichkeiten für engagierte Ingenieurinnen und Ingenieure."

Fakten:

Mit über 6.200 Mitarbeiterinnen und Mitarbeitern ist AVL das weltweit größte unabhängige Unternehmen für die Entwicklung von Antriebssystemen (Verbrennungsmotoren, Hybrid Systeme, elektrische Antriebe) sowie Simulation und Prüftechnik für PKW, LKW und Großmotoren. 2012 erzielte das Unternehmen einen Umsatz von 1.015 Mio. Euro, weltweit hat AVL 45 Niederlassungen.

Wenn Sie Karriere bei AVL machen wollen, freuen wir uns über Ihre Bewerbungsunterlagen unter www. avl.com/jobs 